\title{
Preface to Volume 2020, Special Issue on Designs for the NIST Lightweight Standardisation Process
}

\author{
Itai Dinur $^{1}$ and Gaëtan Leurent ${ }^{2}$ \\ ${ }^{1}$ Ben-Gurion University, Beer Sheva, Israel \\ ${ }^{2}$ Inria, Paris, France
}

IACR Transactions on Symmetric Cryptology (ToSC) is a forum for original results in all areas of symmetric cryptography, including the design and analysis of block ciphers, stream ciphers, encryption schemes, hash functions, message authentication codes, (cryptographic) permutations, authenticated encryption schemes, cryptanalysis and evaluation tools, and security issues and solutions regarding their implementation.

This special issue is dedicated to second-round candidates in NIST's (National Institute of Standards and Technology) ongoing standardisation process for lightweight cryptography. This process started with a call for algorithms that received 57 submissions in February 2019, and 32 of them advanced to the second round in August 2019 ${ }^{1}$. One of the main goals of this issue was to encourage designers to submit high-quality schemes which might otherwise not have been formally published. It is the first special issue of ToSC dedicated to a specific topic.

As all ToSC issues, this special issue strives to maintain a high quality review process and a high quality of published articles. Full papers are assigned to at least three members of the Editorial Board; for submissions by Editorial Board members this was increased to at least four. These members write detailed and careful reviews (usually without relying on subreviewers). Moreover, we have had a rebuttal phase, allowing authors to respond to the review comments before the final decisions. If necessary, the review process enables further interactions between the authors and the reviewers, mediated by the Co-Editors-in-Chief.

Overall, we are very pleased with the quality and quantity of submissions, the detailed review reports written by the reviewers and the substantial efforts by the authors to further improve the quality of their work. We think that the review process, and in particular the use of major revisions, leads to an increased quality of the papers that are published.

For this special issue we received 17 submissions, out of which 12 were accepted, 8 of those after a minor revision, and 1 of those after a major revision.

We would like to thank the authors of all submissions for contributing high quality submissions. In particular, we would like to thank the Editorial Board members; we value their hard work and dedication to write constructive and detailed reviews and to engage in interesting discussions. Many Editorial Board members spent additional time as shepherds to help the authors improving their works.

\footnotetext{
${ }^{1}$ https://csrc.nist.gov/Projects/lightweight-cryptography/round-2-candidates
} 
We also would like to thank Anne Canteaut, Shai Halevi, Gregor Leander, Friedrich Wiemer, and Phil Hebborn for their work and support. We hope that the papers in this volume of IACR Transactions on Symmetric Cryptology (ToSC) prove valuable and we are glad to see that ToSC is becoming the leading international venue publishing the top research on symmetric cryptology.

May 2020

Itai Dinur

Gaëtan Leurent 


\section{Editorial Board}

Frederik Armknecht

Tomer Ashur

Subhadeep Banik

Zhenzhen Bao

Christof Beierle

Christina Boura

Anne Canteaut

Joan Daemen

Patrick Derbez

Christoph Dobraunig

Orr Dunkelman

Maria Eichlseder

Takanori Isobe

Jérémy Jean

Pierre Karpman

Stefan Kölbl

Virginie Lallemand

Jooyoung Lee

Stefan Lucks

Atul Luykx

Willi Meier

Florian Mendel

Bart Mennink

Brice Minaud

Kazuhiko Minematsu

Nicky Mouha

Samuel Neves

Léo Perrin

Thomas Peyrin

Bart Preneel

Yu Sasaki

Hadi Soleimany

Ling Song

Francois-Xavier Standaert

Siwei Sun

Elmar Tischhauser
University of Mannheim, Mannheim, Germany

KU Leuven, Leuven, Belgium

TU Eindhoven, Eindhoven, The Netherlands

Ecole Polytechnique Federale de Lausanne (EPFL), Lausanne, Switzerland

Nanyang Technological University (NTU), Singapore, Singapore

Ruhr University Bochum, Bochum, Germany

University of Versailles, Versailles, France

Inria, Paris, France

Radboud University, Nijmegen, The Netherlands

University of Rennes, Rennes, France

Centre national de la recherche scientifique (CNRS), Rennes, France

Institut de Recherche en Informatique et Systèmes Aléatoires (IRISA), Rennes, France

Radboud University, Nijmegen, The Netherlands

University of Haifa, Haifa, Israel

Graz University of Technology, Graz, Austria

University of Hyogo, Kobe, Japan

Agence Nationale de la Sécurité des Systèmes d'Information (ANSSI), Paris, France

Université Grenoble Alpes, Grenoble, France

Google, Zurich, Switzerland

Centre National de la Recherche Scientifique (CNRS) Nancy, France

Korea Advanced Institute of Science and Technology (KAIST), Daejeon, South Korea

Bauhaus-Universität Weimar, Weimar, Germany

Swirlds Inc., United States

University of Applied Sciences and Arts Northwestern Switzer-

land (FHNW), Windisch, Switzerland

Infineon Technologies, Munich, Germany

Radboud University, Nijmegen, The Netherlands

Inria, Paris, France

École Normale Supérieure (ENS), Paris, France

NEC, Kawasaki, Japan

National Institute of Standards and Technology (NIST),

Gaithersburg, United States

University of Coimbra, Coimbra, Portugal

Inria, Paris, France

Nanyang Technological University (NTU), Singapore,

Singapore

KU Leuven, Leuven, Belgium

NTT Secure Platform Laboratories, Tokyo, Japan

Shahid Beheshti University, Teheran, Iran

Nanyang Technological University (NTU), Singapore

Chinese Academy of Sciences, Beijing, China

UCLouvain, Louvain-la-Neuve, Belgium

Chinese Academy of Sciences, Beijing, China

Philipps-Universität Marburg, Marburg, Germany 
Yosuke Todo

Gilles Van Assche

Damian Vizár
NTT Secure Platform Laboratories, Tokyo, Japan

STMicroelectronics, Diegem, Belgium

Centre suisse d'électronique et de microtechnique (CSEM), Neuchâtel, Switzerland

\section{External reviewers}

Vasily Mikhalev 